\title{
Bound states between dark matter particles, and emission of gravitational radiation
}

\author{
Grigoris Panotopoulos \\ ASC, Physics Department LMU, \\ Theresienstr. 37, 80333 Munich, Germany \\ email:Grigoris.Panotopoulos@physik.uni-muenchen.de
}

\begin{abstract}
Bound states of two weakly interactive massive particles are studied. It is assumed that the WIMPonium is formed due to the gravitational interaction, since the weak interaction can sometimes be repulsive. The lifetimes of the spontaneous emission of gravitational radiation and of the WIMPs annihilation into a pair of gravitons are computed, and are shown to be many orders of magnitude larger than the age of the universe.
\end{abstract}




\section{Introduction}

There is accumulated evidence both from astrophysics and cosmology that about $1 / 4$ of the energy budget of the universe consists of so called dark matter, namely a component which is non-relativistic and does not feel the electromagnetic nor the strong interaction. For a review on dark matter see e.g. [1. Although the list of possible dark matter candidates is long (for a nice list see e.g. [2]), it is fair to say that the most popular dark matter particle is the LSP in supersymmetric models with R-parity conservation [3]. The superpartners that have the right properties for playing the role of cold dark matter in the universe are the axino, the gravitino and the lightest neutralino. By far the most discussed case in the literature is the case of the neutralino (see the classical review [4]), probably because of the prospects of possible detection. Up to now the nature of the cold dark matter particle is unknown. Several indirect detection experiments (see e.g. [5]) are looking for annihilation products of dark matter in astrophysical contexts. Recently there has been some interest in annihilation of WIMP dark matter via intermediate long-lived WIMPonium bound states, which can lead to potentially very substantial enhancements in the dark matter annihilation rate [6]. In these works the authors have considered the case in which the WIMPonium bound states are formed due to the weak interaction. This may sound as the only possibility, since in particle physics gravity is usually ignored, and WIMPs do not interact neither strongly nor electromagnetically. However, it is known from field theory [7] that if an interaction is mediated by a spin one gauge boson it can be either attractive or repulsive, and more presicely the force between two identical particles (e.g. electron-electron or positron-positron) is repulsive, while the force between non-identical particles (e.g. electron-positron) is attractive. In the present work we wish to study the case in which WIMPonium is formed because of the gravitational interaction. It is interesting in this case that everything can be computed explicitly, and there are certain expressions for probability rates, cross sections, lifetimes etc. As a limited case of applicability, one could have in mind the neutralino case (majorana fermions).

A remark is in order here. We shall be considering a framework in which the weak interaction cannot bind WIMPs together to form WIMPonium. However, the weak interaction can still mediate transitions and annihilations that most likely dominate over the gravitational ones computed in the next sections of this article. Therefore, in reality we shall be studying a case of 
academic interest in which the gravitational interactions for the aforementioned processes dominate for a WIMP with no weak interactions at all, and not a generic WIMP or neutralino.

Our work is organized as follows. The present article consists of three sections. After this introductory section, we present the analysis and our results in section 2 , and finally we conclude in the last section.

\section{Analysis}

This is the main section of the present article. In the first subsection we recall the quantum mechanical problem for the $1 / r$ potential case, then we compute the transition rate between two arbitrary bound states A and B, and finally in the third subsection we compute the WIMPonium lifetime due to WIMPs annihilation into a pair of gravitons.

\subsection{Energy levels and wavefunctions}

In the non-relativistic limit the two WIMPs (particle-antiparticle) system is described by the Hamiltonian

$$
H=\frac{p_{1}^{2}}{2 M}+\frac{p_{2}^{2}}{2 M}+V(r)
$$

where $M$ is the WIMP mass, $p_{i}, 1=1,2$ are the momentum operators, and $V(r)$ is the gravitational potential given by

$$
V(r)=-G \frac{M^{2}}{r} \equiv \frac{\alpha_{g}}{r}
$$

with $r$ being the distance between the particle and its antiparticle. Ignoring the center-of-mass motion, which is that of a free particle with mass $2 M$, the dynamics of the two-body system is described by the Hamiltonian

$$
h=\frac{p^{2}}{2 \mu}+V(r)
$$

where $\mu=M / 2$ is the reduced mass of the system, and $p$ is the momentum operator conjugate to the position operator $r$. Because of the spherical symmetry it is convenient to use the spherical coordinate system, in which case one has to solve a one-dimensional problem with respect to $r$

$$
y^{\prime \prime}(r)+2 \mu\left(E-V_{e f f}(r)\right)=0
$$


where the effective potential is given by

$$
V_{e f f}=V(r)+\frac{l(l+1)}{r^{2}}
$$

with $l$ being the angular momentum quantum number. Finally, the wavefunction is given by

$$
\Psi_{n l m}(r, \theta, \phi)=Y_{l}^{m}(\theta, \phi) \frac{y_{n l}(r)}{r}
$$

with $Y_{l}^{m}$ the spherical harmonics, and with the normalization conditions

$$
\begin{aligned}
& 1=\int d \Omega\left|Y_{l}^{m}\right|^{2} \\
& 1=\int_{0}^{\infty} d r|y|^{2}
\end{aligned}
$$

while the allowed energy levels are given by

$$
E_{n}=\frac{E_{1}}{n^{2}}
$$

where $n$ is the principal quantum number, and $E_{1}$ is the energy corresponding to the ground state. Later on we shall make use of $E_{1}, a_{0}$, and therefore we report here their values

$$
\begin{aligned}
a_{0} & =\frac{1}{\mu \alpha_{g}} \\
E_{1} & =-\frac{\mu \alpha_{g}^{2}}{2}
\end{aligned}
$$

or equivalently

$$
\begin{aligned}
a_{0} & =2\left(\frac{m_{p l}}{M}\right)^{3} l_{p l} \\
E_{1} & =-\frac{1}{4}\left(\frac{M}{m_{p l}}\right)^{4} M
\end{aligned}
$$

where $m_{p l} \sim 10^{19} \mathrm{GeV}$ is the Planck mass, and $l_{p l} \sim 10^{-33} \mathrm{~cm}$ is the Planck length. 


\subsection{Spontaneous emission of gravitational radiation in the quadrupole approximation}

In the case of spontaneous emission, a WIMPonium state A makes a transition to a state $\mathrm{B}$ in the absence of any incident gravitational wave. In this subsection we shall derive the lifetime of the state A by exploiting the similarities with the electromagnetic theory. Let us first recall here that in atomic transitions the dominant contribution (whenever is allowed by the selection rules) in the spontaneous emission of electromagnetic radiation is the transition in the electric dipole approximation. In the quantum theory of radiation one obtains the following expression for the integrated transition probability between two atomic states A and B [8]

$$
\Gamma_{A->B}=\frac{e^{2} \omega^{3}}{3 \pi}\left|\vec{x}_{B A}\right|^{2}
$$

or

$$
\Gamma_{A->B}=\frac{\omega^{3}}{3 \pi}\left|\vec{p}_{B A}\right|^{2}
$$

where $e$ is the electron charge, $\omega$ is the energy of the photon emitted, $\vec{x}_{B A}=$ $\langle B|\vec{x}| A\rangle$ is the matrix element of the position operator between the states $\mathrm{A}$ and $\mathrm{B}$, and we have defined $\vec{p} \equiv e \vec{x}$. On the other hand, in the classical electromagnetic theory the average power for the dipole radiation is given by [9]

$$
\bar{P}=\frac{p_{0}^{2} \omega^{4}}{12 \pi}
$$

where $p_{0}$ is the amplitude of the oscillatory electric dipole moment of the radiating source, $p(t)=p_{0} \cos (\omega t)$. Remarkably enough, one can use the classical result to obtain the formula for the transition rate $\Gamma_{A->B}$ given above, avoiding the full quantum mechanical computation. Within the time interval $\tau=\Gamma^{-1}$, with $\tau$ being the lifetime of the state A, one photon with energy $\omega$ is emitted, and therefore we can write

$$
\bar{P}=\frac{\omega}{\tau}=\omega \Gamma
$$

Therefore, the transition rate $\Gamma$ is determined and given by

$$
\Gamma=\frac{\bar{P}}{\omega}=\frac{\omega^{3}}{3 \pi}\left|\frac{p_{0}}{2}\right|^{2}
$$


which means that from the classical result, the quantum mechanical result is obtained provided that we replace $p_{0} / 2$ by the matrix element $\vec{p}_{B A}$. This is not strange at all, if we recall that in the classical treatment the radiating source is characterized by an oscillatory dipole moment $p(t)=p_{0} \cos (\omega t)$, while in the quantum theory of radiation the interaction Hamiltonian is written in the form $H_{I}(t)=H_{I}^{\prime} \exp ( \pm i \omega t)$. Therefore, if we write the $\cos (\omega t)$ as a sum of two exponentials, $\cos (\omega t)=(1 / 2)(\exp (i \omega t)+\exp (-i \omega t))$, we recover the substitutional rule $p_{0} / 2 \rightarrow \vec{p}_{B A}$.

Now let us move to the gravitational radiation case. It is known [10] that in the classical theory of general relativity the emission of radiation due to electric or magnetic dipole vanishes. The first non-vanishing contribution comes from the quadrupole emission mechanism. So, we need the classical result for the power for gravitational radiation in the quadrupole approximation, and then we can easily obtain the corresponding quantum mechanical result. In the classical electromagnetic theory, the power for radiation in the quadrupole approximation is given by [11]

$$
P_{E M}=\frac{k_{c} \omega^{6}}{360} \sum_{\alpha, \beta}\left|Q_{\alpha \beta}\right|^{2}
$$

where $k_{c}=1 /\left(4 \pi \epsilon_{0}\right)$ is the constant entering into Coulomb's law for the electric force between two charges, and the quadrupole tensor $Q_{\alpha \beta}$ is defined as

$$
Q_{\alpha \beta}=\int d^{3} \vec{x} \rho(\vec{x})\left(3 x_{\alpha} x_{\beta}-|\vec{x}|^{2} \delta_{\alpha \beta}\right)
$$

with $\rho(\vec{x})$ being the electric charge density with the property

$$
\int d^{3} \vec{x} \rho(\vec{x})=Q_{T O T}
$$

where $Q_{T O T}$ is the total electric charge in space. The classical result for gravitational radiation in the quadrupole radiation looks like the one for electromagnetic radiation

$$
P_{G}=\frac{4 G \omega^{6}}{360} \sum_{\alpha, \beta}\left|I_{\alpha \beta}\right|^{2}
$$

where now $k_{c}$ is replaced by the Newton's constant $G$, the extra numerical factor of 4 is due to tensor calculus [10], and $I_{\alpha \beta}$ is the gravitational 
counterpart of $Q_{\alpha \beta}$. Now we have the mass density with the property

$$
\int d^{3} \vec{x} \rho_{M}(\vec{x})=M_{T O T}
$$

with $M_{T O T}$ being the total mass in space, and the mass quadrupole tensor

$$
I_{\alpha \beta}=\int d^{3} \vec{x} \rho_{M}(\vec{x})\left(3 x_{\alpha} x_{\beta}-|\vec{x}|^{2} \delta_{\alpha \beta}\right)
$$

and for the WIMPonium an order-of-magnitude estimation is

$$
I_{\alpha \beta} \sim M a_{0}^{2}
$$

Finally, by applying the rule $I_{\alpha \beta} / 2 \rightarrow I_{B A}^{\alpha \beta}$ we obtain the transition rate between two WIMPonium states A and B in the quadrupole approximation

$$
\Gamma_{A \rightarrow B}^{G r}=\frac{2 G \omega^{5}}{45} \sum_{\alpha, \beta}\left|I_{B A}^{\alpha \beta}\right|^{2}
$$

where the matrix element $I_{B A}^{\alpha \beta}$ reads

$$
I_{B A}^{\alpha \beta}=\left\langle B\left|I_{\alpha \beta}\right| A\right\rangle
$$

Taking into account that $G=1 / m_{p l}^{2}, \omega \sim\left|E_{1}\right|$, and $\left\langle B\left|I_{\alpha \beta}\right| A\right\rangle \sim M a_{0}^{2}$, we finally obtain the formula

$$
\tau_{A->B} \simeq\left(\frac{m_{p l}}{M}\right)^{15} t_{p l}
$$

where $t_{p l} \sim 10^{-44}$ sec is the Planck time. Assuming a WIMP mass $M=$ $10 \mathrm{TeV}$ we find for the lifetime

$$
\tau_{A->B} \simeq 10^{163} t_{0}
$$

with $t_{0}$ being the age of the universe, and we have used the numerical values

$$
\begin{aligned}
t_{0} & \simeq 10^{10} \mathrm{yr} \\
y r & \simeq 3 \times 10^{7} \mathrm{sec}
\end{aligned}
$$




\subsection{WIMPonium ground state annihilation into a pair of gravitons}

We assume that the WIMP particles responsible for the cold dark matter in the universe are scalar fields. The available data do not require that WIMPs must be fermions, and as a matter of fact there are already in the literature scalar field candidates for playing the role of dark matter. We can mention at least the case of the axion [12, which has been introduced in order to solve the strong CP problem via the Peccei-Quinn mechanism [13], and the case of branons that are related to the fluctuations of a D-brane [14 on which we are confined according to the brane-world idea [15]. We choose to work with scalar fields to avoind extra complications from spinorial fields, but our final results should also hold for fermionic WIMPs, since we eventually take the non-relativistic limit.

First let us recall how one computes the annihilation rate of the positronium ground state into a pair of photons. Ignoring for one moment that we have a bound state, one needs to compute the total unpolarized cross section of the process, $e^{-} e^{+} \rightarrow \gamma \gamma$, using standard field-theoretic techniques, then take the non-relativistic limit, and finally take into account that what we have is really a bound state. For that we also need the ground state wavefunction, which has already been determined by solving the Schoedinger's equation. All in all, the inverse of the positronium lifetime (ground state) is given by [8]

$$
\Gamma\left(n=1,{ }^{1} S \rightarrow 2 \gamma\right)=4 \sigma_{\text {tot }}^{\text {unpol }} v\left|\Psi_{1 s}(\vec{x}=0)\right|^{2}
$$

where the factor of 4 is due to the spin, namely we have taken into account that in the positronium ground state the single state does the whole job.

Now let us move to the graviton-WIMPs system. According to general relativity, if we have a canonical scalar field coupled to gravity, then the total action describing the model contains the Einstein-Hilbert term for the gravity part plus the appropriate term for the scalar field

$$
S=\int d^{4} x \sqrt{-g}\left(-\frac{R}{2 \kappa^{2}}+\frac{1}{2}(\partial \phi)^{2}-V(\phi)\right)
$$

where $R$ is the Ricci scalar, $g$ is the determinant of the metric, $\kappa=\sqrt{8 \pi G}$ is the strength of the gravitational interaction, and $V(\phi)$ is the self-interacting potential for the scalar field. For the discussion to follow we shall consider the case of a massive scalar field, $V(\phi)=(1 / 2) M^{2} \phi^{2}$. If we write the metric 
as $g_{\mu \nu}=\eta_{\mu \nu}+\kappa h_{\mu \nu}$, it possible to obtain a usual field theory in Minskowski spacetime, the "scalar gravitodynamics", which describes a massless spin two particle (graviton), a massive spin zero particle (WIMP) interacting with each other. The total Lagrangian takes the form [16]

$$
\begin{aligned}
S & =\int d^{4} x \mathcal{L} \\
\mathcal{L} & =\mathcal{L}\left[h_{\mu \nu}\right]+\mathcal{L}[\phi]-\frac{\kappa}{2} h^{\mu \nu} T_{\mu \nu}+\mathcal{L}[h h h]+\ldots
\end{aligned}
$$

where the first term is for free gravitons, the second term is for free WIMPs, the third term is responsible for the interaction between the gravitons and WIMPs, and the rest of the Lagrangian describes the interaction of gravitons among themselves. Here $T_{\mu \nu}$ is the energy-momentum tensor for a scalar field

$$
T_{\mu \nu}=\partial_{\mu} \phi \partial_{\nu} \phi-\left(\frac{1}{2}(\partial \phi)^{2}-V(\phi)\right) g_{\mu \nu}
$$

Given this interaction term one can derive the Feynman rules for the interaction vertices of this model [16]. Regarding the free part of the total Lagrangian, one needs to quantize the free scalar field, as well as the free graviton. The scalar field quantization is well established in many standard books on field theory. As for the graviton case, the treatment should be similar to quantum electrodynamics. There are a few differences due to the fact that the photon field carries a single Lorentz index $\left(A^{\mu}\right.$, spin one boson, polarization vector $\left.\epsilon_{\lambda=1,2}^{\mu}(k)\right)$, while the graviton carries two Lorentz indices $\left(h^{\mu \nu}\right.$, spin two boson, polarization vector $\left.\epsilon_{\lambda=1,2}^{\mu \nu}(k)\right)$. Finally, the Feynman propagator in momentum space for a free graviton reads (in the commonly used De Donder gauge) [17]

$$
\begin{aligned}
D_{F}^{\mu \nu \alpha \beta}(k) & =\frac{P^{\mu \nu \alpha \beta}}{k^{2}-i \epsilon} \\
P^{\mu \nu \alpha \beta} & =\frac{1}{2}\left(\eta^{\mu \alpha} \eta^{\nu \beta}+\eta^{\mu \beta} \eta^{\nu \alpha}-\eta^{\mu \nu} \eta^{\alpha \beta}\right)
\end{aligned}
$$

In a similar way to the electromagnetic case and the positronium, we are now interested in the process, $\phi \phi \rightarrow G G$, with $G$ being the graviton, and we first need to obtain the total unpolarized cross section at tree level and in the non-relativistic limit. Then we can obtain the lifetime of the WIMPonium ground state taking into account the corresponding wavefunction. For the 
computation of the total cross section for the process $1+2 \rightarrow 3+4$ we could start from the standard expression for the differential cross section in the center-of-mass system given by [8]

$$
\left(\frac{d \sigma}{d \Omega}\right)_{C M}=\frac{1}{64 \pi^{2} s} \frac{\left|\vec{p}_{f}\right|}{\left|\overrightarrow{p_{i}}\right|}|\mathcal{M}|^{2}
$$

where $s$ is the Mandelstam's variable, $\vec{p}_{i}$ and $\vec{p}_{f}$ are the initial and final momenta respectively, and $\mathcal{M}$ is the Feynman amplitude computed by applying the Feynman rules for a given model. Then we could apply Feynman's rules to compute the invariant amplitude $\mathcal{M}$, and finally we could integrate over the solid angle to obtain the total cross section. However, we do not really have to go into this, since we are only interested in the non-relativistic limit, $M \gg K$, with $K$ being the kinetic energy, in which the energy of the WIMP is $E \simeq M$, and the WIMP momentum is $p \simeq M v$. In this limit we can see from eq. (39) that the cross section goes like $\sigma \sim 1 / v$, and from second order perturbation theory and dimensional analysis the cross section should be

$$
\sigma \sim \frac{G^{2} M^{2}}{v}
$$

where the precise numerical prefactor turns out to be $2 \pi$ [18]. Therefore, in total the WIMPonium lifetime is given by (since now there is no extra complication related to the WIMP spin)

$$
\tau_{\text {WIMPonium }}^{-1}=\Gamma_{\text {WIMPonium }}=\sigma_{N R} v|\Psi(0)|^{2}=2 \pi G^{2} M^{2} \frac{1}{\pi a_{0}^{3}}=\frac{1}{4}\left(\frac{M}{m_{p l}}\right)^{10} M
$$

or

$$
\tau_{\text {WIMPonium }}=4\left(\frac{m_{p l}}{M}\right)^{11} t_{p l}
$$

If the WIMP mass is $M=10 \mathrm{TeV}$, then the last formula for the WIMPonium lifetime gives

$$
\tau_{\text {WIMPonium }} \simeq 10^{104} t_{0}
$$

Therefore, according to our numerical results we conclude that the lifetimes (both for WIMP annihilation into a pair of gravitons and for transitions between bound states in the quadrupole approximation) are many orders of magnitude (in fact astronomically large!) larger than the age of the universe. Since we have obtained these results in the non-relativistic limit, we expect the same result to be valid for fermionic dark matter particles, although our discussion has been focused on scalar dark matter particles. 


\section{Conlusions}

In the present work we have considered bound states formed due to the gravitational interaction between two identical weakly interactive massive particles. These particles are the ones responsible for the cold dark matter in the universe, and therefore they no not have neither strong neither electromagnetic interactions. Furthermore, if the particle is identical to its own antiparticle we expect from general field theoretic arguments that the weak interaction between the particle and its antiparticle should be repulsive. In this case a bound state can be formed due to the gravitational interaction only, which is a universally attractive interaction. We have assumed that WIMPs are scalar particles for simplicity, in which case we can easily write down the Lagrangian for the WIMP-graviton system, and determine the Feynman rules for computing certain processes in quantum gravity. We do not worry about the non-renormalizability of quantum gravity, since in this work we are only interested in processes at tree level. In the non-relativistic limit the allowed energy levels and the corresponding wavefunctions of WIMPoniums can be computed exactly, and are known from the familiar Hydrogen atom case. We have computed the lifetime of a bound state due to spontaneous emission of gravitational radiation in the quadrupole approximation, as well as the lifetime of the ground state of WIMPonium due to the WIMPs annihilation to a pair of gravitons. We have determined numerical values for the lifetimes, which are shown to be many orders of magnitude larger than the age of the universe.

\section{Acknowledgments}

We wish to thank the anonymous reviewer for his/her comments and suggestions. This work was supported by project "Particle Cosmology".

\section{References}

[1] C. Munoz, "Dark matter detection in the light of recent experimental results," Int. J. Mod. Phys. A 19 (2004) 3093 arXiv:hep-ph/0309346.

[2] M. Taoso, G. Bertone and A. Masiero, "Dark Matter Candidates: A Ten-Point Test," JCAP 0803 (2008) 022 arXiv:0711.4996 [astro-ph]]. 
[3] J. L. Feng, "Supersymmetry and cosmology," In the Proceedings of 31st SLAC Summer Institute on Particle Physics: Cosmic Connection to Particle Physics (SSI 2003), Menlo Park, California, 28 Jul - 8 Aug 2003, pp L11 arXiv:hep-ph/0405215].

[4] G. Jungman, M. Kamionkowski and K. Griest, "Supersymmetric dark matter," Phys. Rept. 267 (1996) 195 [arXiv:hep-ph/9506380].

[5] S. W. Barwick et al. [HEAT Collaboration], "Measurements of the cosmic-ray positron fraction from $1-\mathrm{GeV}$ to $50-\mathrm{GeV}$," Astrophys. J. 482 (1997) L191 arXiv:astro-ph/9703192;

D. J. Thompson, D. L. Bertsch and R. H. . O'Neal, "The highestenergy photons seen by the Energetic Gamma Ray Experiment Telescope (EGRET) on the Compton Gamma Ray Observatory," arXiv:astro-ph/0412376;

F. Aharonian [HESS Collaboration], "A search for a dark matter annihilation signal towards the Canis Major overdensity with H.E.S.S," arXiv:0809.3894 [astro-ph];

O. Adriani et al. [PAMELA Collaboration], "An anomalous positron abundance in cosmic rays with energies 1.5.100 GeV," Nature 458 (2009) 607 [arXiv:0810.4995 [astro-ph]].

[6] J. D. March-Russell and S. M. West, "WIMPonium and Boost Factors for Indirect Dark Matter Detection," Phys. Lett. B 676 (2009) 133 arXiv:0812.0559 [astro-ph]];

W. Shepherd, T. M. P. Tait and G. Zaharijas, "WIMPonium," arXiv:0901.2125 [hep-ph].

[7] Michael E. Peskin, and Daniel V. Schroeder, "An Introduction to Quantum Field Theory," Westview Press.

A. Zee, "Quantum Field Theory in a Nutshell," Princeton University Press.

[8] Sakurai Jun J, "Advanced quantum mechanics," Addison-Wesley Publishing Company.

[9] David J. Griffiths, "Intoduction to Electrodynamics," Prentice Hall, Upper Saddle River, NewJersey 07458.

[10] Charles W. Misner, Kip S. Thorn, and John Archibald Wheeler, "Gravitation," New York, Freeman. 
[11] Jackson John David, "Classical electrodynamics," John Wiley and Sons.

[12] S.Weinberg, "A New Light Boson?," Phys. Rev. Lett. 40 (1978) 223;

F.Wilczek, "Problem Of Strong P And T Invariance In The Presence Of Instantons," Phys. Rev. Lett. 40 (1978) 279.

[13] R.D.Peccei and H.R.Quinn, "CP Conservation In The Presence Of Instantons," Phys. Rev. Lett. 38 (1977) 1440;

R.D.Peccei and H.R.Quinn, "Constraints Imposed By CP Conservation In The Presence Of Instantons," Phys. Rev. D 16 (1977) 1791.

[14] J. Alcaraz, J. A. R. Cembranos, A. Dobado and A. L. Maroto, 'Limits on the brane fluctuations mass and on the brane tension scale from electron positron colliders," Phys. Rev. D 67 (2003) 075010 arXiv:hep-ph/0212269];

J. A. R. Cembranos, A. Dobado and A. L. Maroto, "Brane-world dark matter," Phys. Rev. Lett. 90 (2003) 241301 [arXiv:hep-ph/0302041];

J. A. R. Cembranos, A. Dobado and A. L. Maroto, "Cosmological and astrophysical limits on brane fluctuations," Phys. Rev. D 68 (2003) 103505 arXiv:hep-ph/0307062.

[15] N. Arkani-Hamed, S. Dimopoulos and G. R. Dvali, "The hierarchy problem and new dimensions at a millimeter," Phys. Lett. B 429 (1998) 263 arXiv:hep-ph/9803315];

I. Antoniadis, N. Arkani-Hamed, S. Dimopoulos and G. R. Dvali, "New dimensions at a millimeter to a Fermi and superstrings at a TeV," Phys. Lett. B 436 (1998) 257 arXiv:hep-ph/9804398.

[16] Richard P. Feynman, "Feynman lectures on gravitation," AddisonWesley Publishing Company.

[17] H. van Dam and M. J. G. Veltman, Nucl. Phys. B 22 (1970) 397.

[18] B. S. DeWitt, "Quantum theory of gravity. III. Applications of the covariant theory," Phys. Rev. 162 (1967) 1239. 